\title{
Antimicrobial Activity of Essential Oils: A 1976-1986 Literature Review. Aspects of the Test Methods
}

\author{
A. M. Janssen ${ }^{1}$, J. J. C. Scheffer ${ }^{1,2}$, and A. Baerheim Svendsen ${ }^{1}$
}

Received: November 30, 1986

\begin{abstract}
The data given in the literature published during 1976-1986 concerning antimicrobial activities of essential oils are treated from an experimental point of view and with regard to a possible practical application. Attention is paid to four factors which are important when testing essential oils: the assay technique; the growth medium; the microorganism; the essential oil.
\end{abstract}

\section{Introduction}

During the last decade (1976-1986), a large number of studies has been performed concerning the antimicrobial activity of essential oils. Testing and evaluation of the antimicrobial activity of essential oils is difficult because of their volatility, their water (in-)solubility, and their complexity. Four factors are especially important when testing essential oils: the assay technique; the growth medium; the micro-organism; the essential oil. These factors will be discussed in separate sections.

\section{The Assay Technique}

The commonly used assay techniques can be classified by the fact whether or not they require a homogeneous dispersion in water. A technique which does not require a homogeneous dispersion in water is the agar overlay technique using:

- discs, holes, or cylinders as the reservoir;

- discs as the source of vapour.

The reservoir containing the essential oil to be tested is brought into contact with an inoculated medium and, after incubation, the diameter of the clear zone around the reservoir (inhibition diameter) is measured. This method was originally designed to monitor the amounts of antibiotic substance in crude extracts. The theory for the agar overlay technique has been described in detail $(1,2)$. Some of the conditions in the proposed model have been checked for their validity in the case of essential oils (3). Because all conditions cannot always be fulfilled, predictions are unreliable when using the proposed model.

Different types of reservoirs have been used: filter paper discs or cylinders placed on the surface of the medium, and holes punched in the medium. In all cases, the amount of oil and the diameter of the reservoir are important parameters. In some studies, the amount of oil adhering to a filter paper disc was estimated (Table I). The amounts reported are quite coherent. Irrespective of whether a precisely measured volume of oil was applied to a disc or the disc was soaked in the oil, the amounts adhering to the disc did not differ (3). In addition, no

\footnotetext{
${ }^{1}$ Division of Pharmacognosy, Center for Bio-Pharmaceutical Sciences, Leiden University, Gorlaeus Laboratories, P.O. Box 9502, NL-2300 RA Leiden, The Netherlands.

2 Address for correspondence.
}

Table I. Essential oil amounts reported to adhere to filter paper discs.

\begin{tabular}{|c|c|c|}
\hline $\begin{array}{l}\text { Diameter of } \\
\text { paper disc }\end{array}$ & Amount & Reference \\
\hline $4 \mathrm{~mm}$ & $3.3 \mathrm{mg}$ & (4) \\
\hline $5 \mathrm{~mm}$ & $4-10 \mu \mathrm{l}$ & (5) \\
\hline $5 \mathrm{~mm}$ & $2 \mathrm{mg}$ & $(6,7)$ \\
\hline $6 \mathrm{~mm}$ & $2-3 \mu \mathrm{l}$ & (3) \\
\hline $6 \mathrm{~mm}$ & $2.5-5 \mu \mathrm{l}$ & (8) \\
\hline $6 \mathrm{~mm}$ & $20 \mu \mathrm{l}$ & (9) \\
\hline $9.5 \mathrm{~mm}$ & $20 \mu l^{*}$ & (10) \\
\hline
\end{tabular}

* = applied as a $10 \%$ ethanolic solution

difference was found between the amounts of a polar or a nonpolar compound (3).

In the case of cylinders, a certain minimum volume of oil is necessary to observe any inhibition $(11-13)$ and there is an optimum volume beyond which no further increase in inhibition diameter takes place (11-13). However, the significance of these volumes in the description of the antimicrobial activity of an essential oil is not known. When cylinders and discs of similar diameter were used for the same oil, equal inhibition diameters were found (11-13). This is striking because the applied volumes differed widely.

In some studies, dilutions were applied to the reservoir and the dilution showing no inhibition was determined (e.g. 1115). Such "critical dilutions" were compared with the MIC (minimum inhibitory concentration) values of several Origanum and Thymus oils (3). However, the critical dilutions and the MIC values did not show a strict correlation.

Whether or not a correlation between inhibition diameters and MIC values exists has been studied for many essential oils and aroma chemicals (10). Morris et al. (10) stated that: "qualitative Petri dish screening methods and quantitative minimum inhibitory concentration methods are not necessarily comparable".

In order to lower the detection limit, the inoculated system can be kept at a low temperature before incubation, which favours diffusion over microbial growth and thus increases the inhibition diameter. This technique has been used for obtaining biograms (3) and for the testing of essential oils $(9,16)$.

A number of oils have been tested against fungi. Inhibition diameters determined after 2 and 15 days differed markedly for some oils (17), which might be due to decomposition or evaporation of the oils during the test period.

In a modification of the technique, the reservoir is placed in the lid of the Petri dish, thus excluding transport by diffusion (e.g. 18, 19). In one study (19), this vapour phase technique and the agar overlay technique were compared using various oils. Hyssop oil inhibited Sarcina ureae completely in the paper disc technique whereas it was not at all active via the vapour 
phase; on the other hand, marjoram oil caused inhibition of a Micrococcus luteus strain via the vapour phase, but not in the common paper disc technique. Thus, the two techniques can yield different results.

Instead of an essential oil, sometimes crushed plant material was used (20-22). The vapour emanating from various plants was found to inhibit some fungi. Also for plants in nature this could be of importance, viz. to inhibit the growth of phytopathogens. An activity via the vapour phase can be brought about by the direct action of the vapour on the microorganism, but also indirectly, for example by increasing the concentration of ozonides in the air (23).

In most studies, inhibition zones are compared with those obtained for antibiotics. This is useful in order to control the sensitivity of the test organism, but a comparison of the antimicrobial activity of essential oils and antibiotics cannot be made from this!

Techniques which do require a homogeneous dispersion in water are the so-called dilution techniques that are used to determine:

- MIC or MLC (minimum lethal concentration) values;

- growth curves;

- growth inhibiting properties (poisoned food technique);

- the period before fungal growth occurs;

- antiseptic activity.

The physico-chemical properties of the dispersions used are important for the activity to be observed; these properties depend upon the preparation method. Muenzing and Schels (24) used ultrasonic energy. In some studies, an essential oil was mixed with the agar medium which, thereafter, was allowed to solidify (e.g. 25-27). Also, surface-active substances were used. Simeon de Buochberg (28) prepared emulsions with polysorbatum 80 and essential oil in a certain volume ratio, and subsequently used ultrasonic energy. In some other studies, the problem was ignored and an oil was just added to a liquid medium (e.g. 9, 29-31).

Although the addition of an emulsifying agent introduces an extra component with respect to activity and possible interactions, the method has shown good reproducibility (28). For the other techniques, including the diffusion tests, reproducibility has not been studied.

In a homogeneous system, various assay techniques can be applied. Most often MIC or MLC values are determined. The principles of the technique have been described by Kavanagh (32). The MIC values can be determined either in a liquid or in a solid medium, the MLC values only in a liquid medium. The term MID (maximum inhibitory dilution) has also been employed (33) because usually volume over volume percentages are used.

Use of a homogeneous system also allows growth curves of a micro-organism to be recorded. In general, the turbidity is taken as an indication of bacterial density (e.g. 9, 28, 31). In every case, the growth curves are compared with those obtained in a medium without essential oil. Differences with respect to the lag time, the growth rate, and the maximum density attained are seen. Growth curves for six chemotypes of Thymus vulgaris were recorded (28). For all oil types, the lag times for Escherichia coli and Staphylococcus aureus increased with increasing concentrations of oil. However, no decrease of the growth rate in the exponential phase was seen and all curves eventually attained the same maximum density. In another study (9), the growth curves were not sufficiently neat to allow the calculation of lag times and/or growth rates. Citral markedly inhibited the growth of Escherichia coli at a concentration of $0.05 \%(\mathrm{v} / \mathrm{v})$, but the maximum densities measured for the test substances varied to a certain extent.

The poisoned food technique is used to test essential oils against fungi (e.g. 34-36). A mycelial disc of the test fungus is placed at the centre of a Petri dish containing the substance to be tested in a solid medium. Growth of the disc is compared with that of a mycelial disc placed on a medium without test substance. In addition, by cultivating the disc on fresh medium, fungicidal and fungistatic activity can be distinguished. Solid medium containing the test substance is also used for another experiment. The medium is inoculated with a fungus and the period until macroscopic growth occurs is compared with that of a blank experiment.

Disinfectant activity can be determined by measuring the period necessary to kill a small inoculum of bacteria. This interval can be correlated with the concentration of the disinfectant (37). Whether or not this correlation holds true for essential oils has not been verified.

\section{The Growth Medium}

The medium, i.e. the direct environment of the test organism, is an important factor in determining the antimicrobial activity of a substance. Medium constituents may react with essential oil components and activate or inactivate them. Thus, it is known that some aldehydes, such as perillaldehyde, can react with the sulfhydryl groups in proteins (25).

In early studies on the agar overlay technique, the influence of the agar content on the solubility of some oil constituents was established. The higher the agar content, the lower the solubility and, thus, the smaller the inhibition zones (38).

The $\mathrm{pH}$ of the medium seems to play an important role as well. For phenols (39) and carboxylic acids, it is known that only the uncharged form is able to penetrate the microbial cell and to exhibit activity. It was found (40) that the $\mathrm{pH}$ can also have an influence when testing neutral molecules. For Grampositive, non-lactic bacteria the activities of eugenol, $l$-carvone, $d$-carvone, and menthol, respectively, were larger at $\mathrm{pH}$ 6 than at $\mathrm{pH} 8$. Anise oil was more active against Penicillium brevicompactum, Aspergillus fumigatus, and Cladosporium herbarum at $\mathrm{pH} 4.8$ than at $\mathrm{pH} 6.8$ (41). The essential oil of Hyptis suaveolens was most active at $\mathrm{pH} 9$ when tested against Helminthosporium oryzae by the poisoned food technique (42). The oils of Cedrus deodora and Mentha arvensis var.

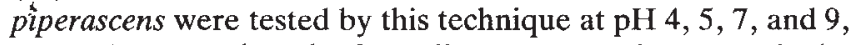
respectively. Cedrus deodora oil was most active at $\mathrm{pH} 9$, the mint oil at $\mathrm{pH} 4$ (43). For some micro-organisms, response differences have been observed even within a range of one $\mathrm{pH}$ unit (44).

\section{The Micro-organism}

The activity measured for related micro-organisms can sometimes be quite different. Even within one species this may be true: the MIC values determined for the essential oils of Thymus vulgaris chemotypes sometimes differed markedly within a chemotype when using different strains of the same micro-organism (28) (Table II). The same phenomenon was observed for various oils using different Streptococcus $D$ strains (19). The strain number of a test organism should therefore be mentioned if possible.

Because the test period for fungi is long, decomposition of oil constituents may occur. However, no attention seems to have been paid to this problem, so far. 
Table II. Range of MIC values $(\mathrm{mg} / \mathrm{ml})$ determined for chemotypes of Thymus vulgaris using different strains of the micro-organisms listed (28).

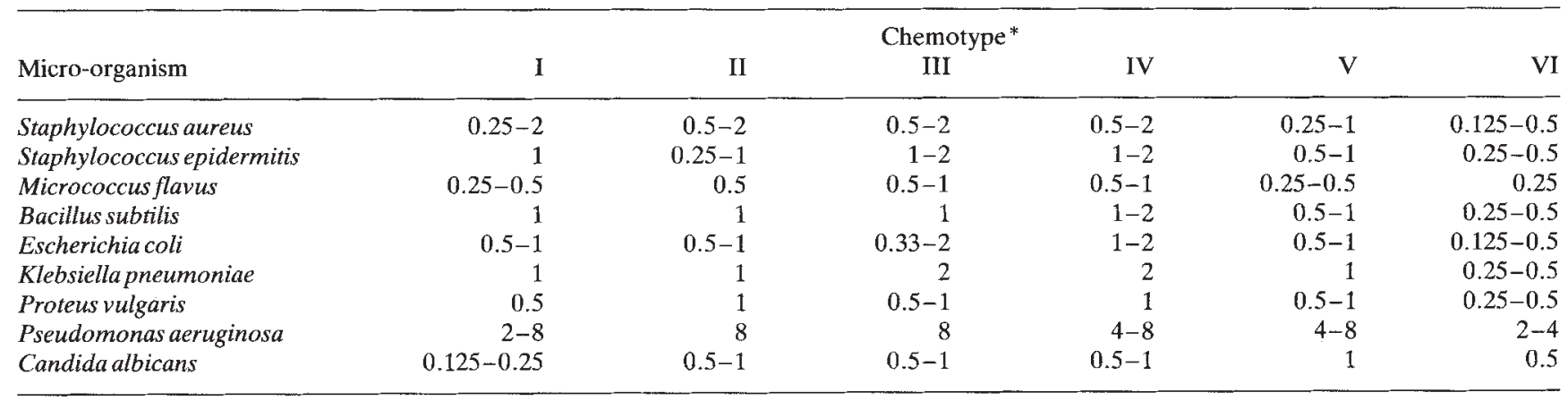

* I: Geraniol type; II: Linalool type; III: $\alpha$-Terpineol type; IV: Thujan-4-ol type; V: Carvacrol type; VI: Thymol type.

\section{The Essential Oil}

Since changes in the composition of an essential oil influence its antimicrobial activity, the following factors should be kept in mind:

- the botanical source (some commercial oils are derived from different species);

- the provenience of the plant material;

- the time of harvesting (time of the day, stage of development);

- the plant material being fresh or dried;

- the isolation technique (steam distillation, hydrodistillation, extraction).

Oil derived from ground, dried rhizomes of Alpinia galanga behaved differently in an agar overlay assay as compared to oil from fresh rhizomes (45).

Kowal et al. (46) found large differences in the MIC and MLC values for essential oils of Thymus pulegioides from different regions in Poland; the time of harvesting proved to be important as well. Oils of Satureja montana from various proveniences yielded different MIC and MLC values when tested against Streptococcus faecalis (47).

Thymus vulgaris and Tanacetum vulgare occur in several chemotypes. The chemotypes of Thymus vulgaris differed mainly in MIC values (28), but for Tanacetum vulgare there were also differences in the activity pattern (48).

Therefore, it is important that the composition of the essential oil tested is given. If this is impossible, one should describe precisely the conditions under which the oil was gained.

\section{Conclusion}

The results given in the literature with regard to the testing of essential oils for antimicrobial activity are difficult to compare. The test methods used differ widely and important factors influencing the results are frequently neglected. Thus, the conclusions drawn in many papers are not based on reproducible experiments. In future studies, one should, for example, mention the strain number of a test organism, if possible, and one should give the essential oil composition or describe precisely under which conditions the oil was gained.

\section{References}

(1) Cooper, K. E. (1963) in: Analytical Microbiology, (Kavanagh, F., ed.), pp. 1-86, Academic Press, New York, London.

(2) Linton, A. H. (1983) in: Antibiotics: Assessment of Antimicrobial Activity and Resistance, (Russell, A. D., Quesnel, L. B., eds.), pp. 19-30, Academic Press, London, New York.

(3) Janssen, A. M., Scheffer, J. J. C., Baerheim Svendsen, A. (1986) in: Progress in Essential Oil Research, (Brunke, E.-J., ed.), pp. 401-419, Walter de Gruyter \& Co., Berlin, New York.

(4) Batra, A., Mehta, B. K. (1985) Fitoterapia 56, 357-- 359.

(5) Gupta, Y. N. (1976) Indian Perfum. 20 Pt. 1a, 45-53.

(6) Murari, N. D., Mathela, C. S. (1978) Indian Perfum. 22, 291-292.

(7) Agarwal, I., Mathela, C. S., Sinha, S. (1979) Indian Phytopathol. 23, 104-105.

(8) Singh, P., Pathak, R. C., Sinha, G. K. (1978) Indian Perfum. 22, $76-78$.

(9) Onawunmi, G. O., Yisak, W., Ogunlana, E. O. (1984) J. Ethnopharmacol. 12, 279-286.

(10) Morris, J. A., Khettry, A., Seitz, E. W. (1979) J. Am. Oil Chem. Soc. 56, 595-603.

(11) Banerjee, A., Nigam, S. S. (1976) Riechst. Aromen Koerperpflegem. 26, 154-155.

(12) Banerjee, A., Nigam, S. S., Kaul, V. K. (1978) Indian Perfum. $22,69-72$.

(13) Banerjee, A., Kaul, V. K., Nigam, S. S. (1978) Indian Perfum. 22, 214-217.

(14) Banerjee, A., Nigam, S. S. (1976) Indian J. Pharm. 38, 103-105.

(15) Tirumala Rao, J. (1976) Riechst. Aromen Koerperpflegem. 26, 50.

(16) Garg, S. C., Tripathi, M. K. (1977) Riechst. Aromen Koerperpflegem. 27, 209.

(17) Yousef, R. T., Tawil, G. G. (1980) Pharmazie 35, 698-701.

(18) Reuveni, R., Fleischer, A., Putievsky, E. (1984) Phytopathol. Z. $110,20-22$.

(19) Pellecuer, J., Jacob, M., Simeon de Buochberg, M., Dusart, G., Attisto, M., Barthez, M., Gourgas, L., Pascal, B., Tomei, B. (1980) Plant, Med. Phytother. 14, 83-98.

(20) Pandey, D. K., Chandra, H., Tripathi, N. N. (1982) Phytopathol. Z. 105, 175-182.

(21) Pandey, D. K., Tripathi, N. N., Tripathi, R. D., Dixit, S. N. (1982) Angew. Bot. 56, 259-267.

(22) Saksena, N., Tripathi, H. H. S. (1985) Fitoterapia 56, 243-244.

(23) Dmitriev, M. T. (1981) in: Fitonsidy: Rol Biogeotsenozakh, Znach. Med., Mater. Soveshch., 8th 1979, (Aizenman, B. E., ed.) pp. 65-73, Izd. Naukova Dumka, Kiev; via (1982) C.A. 97, 97440g.

(24) Muenzing, H. P., Schels, H. (1972) J. Soc. Cosmet. Chem. 23, $841-852$.

(25) Kurita, N., Miyaji, M., Kurane, R., Takahara, Y., Ichimura, K. (1979) Agric. Biol. Chem. 43, 2365-2371.

(26) Szalontai, M., Verzar-Petri, G., Florian, E. (1977) Parfuem. Kosmet. 58, 121-127. 
(27) Singh, A. K., Dikshit, A., Sharma, M. L., Dixit, S. N. (1980) Econ. Bot. 34, 186-190.

(28) Simeon de Buochberg, M., Allegrini, J., Bessière, C., Attisto, M., Passet, J., Granger, R. (1976) Riv. Ital. E.P.P.O.S. 58, 527536.

(29) Knobloch, K., Weigand, H., Weis, N., Schwarm, H.-M., Vigenschow, H. (1986) in: Progress in Essential Oil Research, (Brunke E.-J., ed.) pp. 429-445, Walter de Gruyter \& Co., Berlin, New York.

(30) Bullerman, L. B., Lieu, F. Y., Seier, Sally A. (1977) J. Food Sci. 42, 1107-1109.

(31) Andrews, R. E., Parks, L. W., Spence, K. D. (1980) Appl. Environ. Microbiol. 40, 301-304.

(32) Kavanagh, F. (1963) in: Analytical Microbiology (Kavanagh, F., ed.) pp. 125-141, Academic Press, New York, London.

(33) Villar, A., Recio, M. C., Rios, J. L., Zafra-Polo, M. C. (1986) Pharmazie 41, 298-299.

(34) Dikshit, A., Husain, A. (1984) Fitoterapia 55, 171-176.

(35) Arora, R., Pandey, G. N. (1984) Biol. Mem. 9, 69-72.

(36) Arora, R., Pandey, G. N. (1984) Biol. Mem. 9, 98-104.

(37) Kruchenok, T. B., Lyarskii, P. P., Gleiberman, S. E., Tsetlin, V. M. (1983) Gig. Sanit. 48, 29-33; via (1984) C.A. 100, 79511g.
(38) Schuermann, C., Spitzner, W. (1960) Zentralbl. Bakteriol. Parisitenkd. Infektionskr. Hyg. Abt. 1: Orig. 180, 234-243.

(39) Polster, M., Rittich, B., Zaludova, R. (1986) Collect. Czech. Chem. Commun. 51, 241-248.

(40) Jay, J. M., Rivers, G. M. (1984) J. Food Safety 6, 129-139.

(41) Forstreuter-Kuenstler, M., Ahlert, B. (1984) Lebensmittelchem. Gerichtl. Chem. 38, 143-145.

(42) Pandey, D. K., Tripathi, N. N., Tripathi, R. D., Dixit, S. N. (1982) Z. Pflanzenkr. Pflanzenschutz 89, 344-349.

(43) Dikshit, A., Singh, A. K., Dixit, S. N. (1982) Bokin Bobai 10, $9-$ 10; via (1982) C.A. 96, 149037g.

(44) Laencina, J., Guzman, G., Guevara, L. A., Flores, J. (1985) Essenze Deriv. Agrum. 55, 27-35.

(45) Janssen, A. M., Scheffer, J. J. C. (1985) Planta Med. 507-511.

(46) Kowal, T., Krupinska, A. (1979) Herba Pol. 25, 303-310.

(47) Melegari, M., Albasini, A., Provvisionato, A., Bianchi, A., Vampa, G., Pecorari, P., Rinaldi, M. (1985) Fitoterapia 56, 8591.

(48) Tétényi, P., Héthelyi, E., Kulcser, G., Kaposi, P. (1981) Herba Hung. 20, 57-74. 